\title{
A Method to Detect Breast Cancer Based on Morphological Operation
}

\author{
Prashengit Dhar \\ Department of Computer Science and Engineering, Cox’s Bazar City College, Bangladesh \\ E-mail: nixon.dhar@gmail.com
}

Received: 07 August 2020; Accepted: 05 November 2020; Published: 08 April 2021

\begin{abstract}
Breast cancer is one of the most common cancer in women worldwide. Early detection of breast cancer can lead to better treatment and decrease in mortality. Mammogram image in medical technology, made it easier to analyze breast cancer. Mammography exam is a specialized imaging technique in medical to scan breast which results in mammogram image. Detecting breast cancer earlier, a patient can have several treatment options and also can save live. Early detection of breast cancer can leads to survive 93 percent or greater in the initial five years. This paper proposes a brseast cancer detection method from mammogram image sample by applying morphological operation on gray image rather than binary. Firstly, image is sent for gamma correction. Then it is converted to gray and applied morphological dilation. Again morphological opening operation is formed on the dilated image. Output of dilated and opening operation is then binarized. An AND operation is performed between both binary images. Some post processing likesmall area filtering and hole filling task is took place. Then common unwanted object is removed. Finally rest of the region is the desired cancer infected region. Achieved performance is acceptable and satisfactory through the proposed method.
\end{abstract}

Index Terms: Mammogram image, breast cancer, dilation, opening, detection

\section{Introduction}

Beast cancer is generally considered as a major reason of death due to cancer in women. Both male and female can be suffered from this, but in most of the case it is often found in female over 40 year[2]. It's a challenging task to detect cancer region from mammogram image of breast and also it may not be possible to detect exactly accurate region. However early detection is a necessary step to reduce risk in treatment. A specialized medical imaging named mammography uses low-dose x-ray method to scan the breasts. Mammography exam output which is mammogram image, helps in early detection as well as diagnose too [1]. Screening mammograms are directed to find breast cancer in women without apparent indications. Breast tumors are gradually increases in size with time. A breast tumor generally takes four to five years to reach $1 \mathrm{~mm}$, around two years to reach at $5 \mathrm{~mm}$ and more than one year to be $2 \mathrm{~cm}$ in size $[5,6,7,8]$. The aim of thi research is to locate the cancer region. Using the power of morphological tools, breast cancer detection is presented in this study. Cancer regions can analyzed better in mammogram images. With the presence of nodules, indicate the symptom of cancer. Analyzing mammogram images, provide much information and knowledge about breast cancer region.

In this study, morphological application based breast cancer detection is focused. Dataset named MIAS mammography from kaggle is used in this research [18]. Section III contains an overview of proposed method. Morphological operation involved in this research is discussed in section IV. Complete detection strategy is detailed in section V. Finally, conclusion is in section VI.

\section{Related Works}

For detection, segmentation of image is an effective way to reach at goal. Basically, segmentation partitions an image into several components or objects. Various ways are exists to segment image [3,13]. Double thresholding method for segmentation is a basic way to segment image. [4]. Samir used double thresholding approach for detecting cancer cells in breast [9]. . A mask is applied after performing D-thresh. The whole method also reduces storage area of processing. Mustafa proposed segmentation for breast cancer using GVF snake technique. [11]. Noise is removed using low pass Gaussian filter and then GVF snake technique is applied to find cancer regions. Bacterial foraging technique is used by Dubey for breast cancer detection [10]. It is followed by multi level thresholding. Basheer proposed method to 
segment mass using texture analysis [16]. Also adaptive median filter was employed. The adaptive median filtering helps in contouring image. After that using texture properties of the resultant ROI, required contour is selected.

ML algorithm based detection method is also used by researchers [10]. Abdul proposed SVM algorithm based breast cancer detection method from mammogram images [12]. With the help of texture analysis an SVM, Eddaoudi proposed a method to detect masses. [14]. Sampaio presented a method to detect masses from mammogram image [15]. It removes noise and object beyond boundary. So as internal breast structure is then highlighted. Neural network is used to segment mass. Then shapes are analyzed. Then candidate regions are classified with support vector machine. Wavelet transform and k-means clustering based segmentation is proposed by Dalmiya [17]. Discrete wavelet transform is employed to get max details from MRI images. Sharpness is achieve by adding the output image with original input image. K-means clustering is executed on sharpened image for locating tumor region. Finally, thresholding took place on clustered image to extract tumor region. In this paper, a breast cancer detection method is proposed. Proposed method is mainly based on morphological operations.

\section{System Overview}

This paper presents a system of morphological based breast cancer detection. This section provides a n overview of proposed system. Fig. 1 shows the steps involved in proposed breast cancer detection. Input image is enhanced. For this purpose gamma correction of input is processed and results in an enhanced and suitable form. Dilation task is performed on gamma corrected gray image. Dilation expands contained shape of image. Then morphological opening is performed. Opening is erosion of image and dilation of that eroded image. Binary form of dilated image and opening output is created. Both the binary image is considered for AND operation. Distinctive small areas are filtered .To more fine the image, holes are filled. To get the cancer cell region, common unwanted object located at top is cleared. Rest of the images is considered as cancer cell region as well as breast cancer region. Brief description of these steps is described in section $\mathrm{V}$ with example.

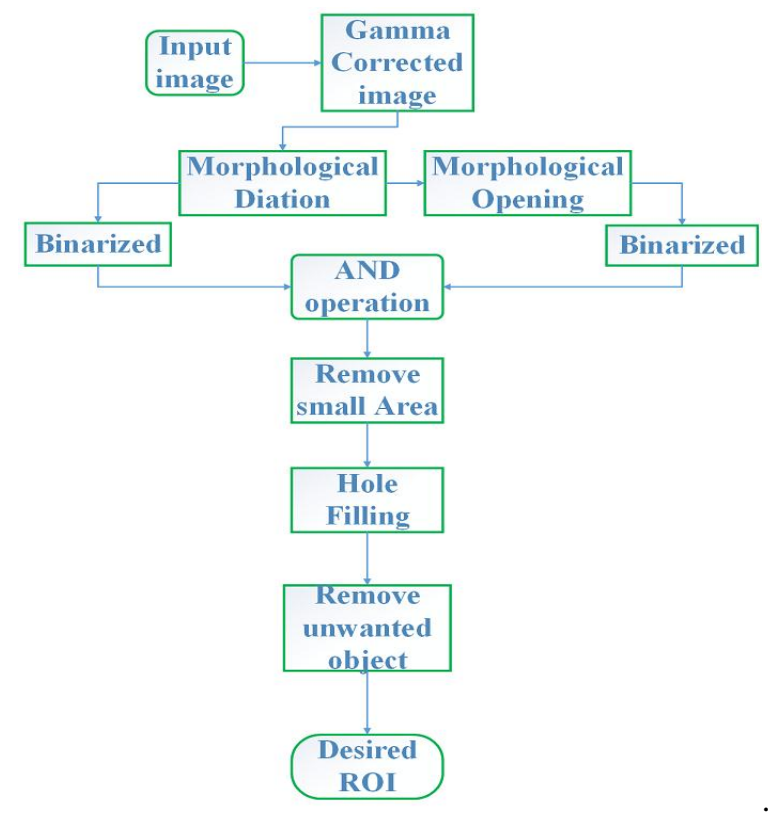

Fig.1. Flow of proposed detection system

\section{Morphlogical Opeartion Overview}

Most common and primary operation of morphological tasks are dilation and erosion. This study considers dilation and opening operation as morphological application. Opening operation is combination of both erosion and dilation. Morphological tasks covers core step in this research.

\section{A. Dilation}

Dilation is a basic morphological operators used in image processing. It is normally functional to binary images, but also works on gray image. The main outcome of the dilation operator while using on binary image is to progressively broaden the boundaries of foreground pixel's (white pixels). region. Therefore, areas of white (foreground) pixels propagate in size while holes lies in those area become smaller. In dilation, the operator takes two 
portions of data in place of inputs. The first one is the image which is needed to be dilated. The another is structuring element (typically small) which is a set of coordinate points. It also known as kernel. The second one i.e structuring element defines the particular effect of the dilation on the image. Fig. 2 is a structuring element of $3 \times 3$ size.

\begin{tabular}{|l|l|l|}
\hline 1 & 1 & 1 \\
\hline 1 & 1 & 1 \\
\hline 1 & 1 & 1 \\
\hline
\end{tabular}

$$
\begin{gathered}
\text { Set of coordinate points }= \\
\{(-1,-1),(0,-1),(1,-1) \\
(-1,0),(0,0),(1,0), \\
(-1,1),(0,1),(1,1)\}
\end{gathered}
$$

Fig.2. Structuring element of size $3 \times 3$
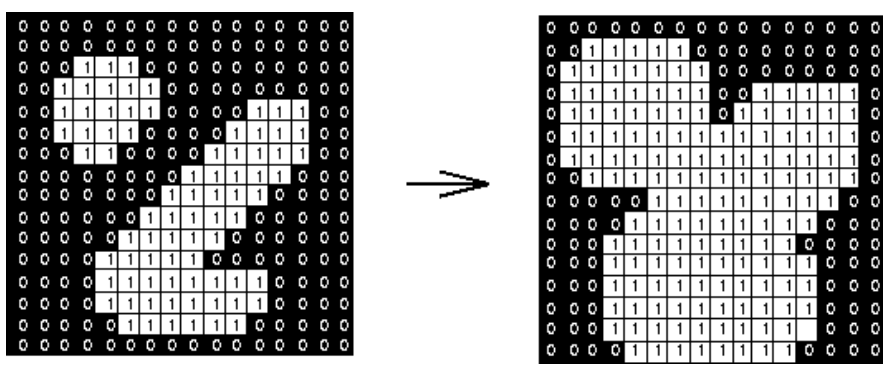

Fig.3. Dilation effect of using $3 \times 3$ structural element

The structuring element of fig.2 is applied on fig.3 (left) for dilation. The result is in fig.3 (right). The dilation operator works like fig.4 on binary image.
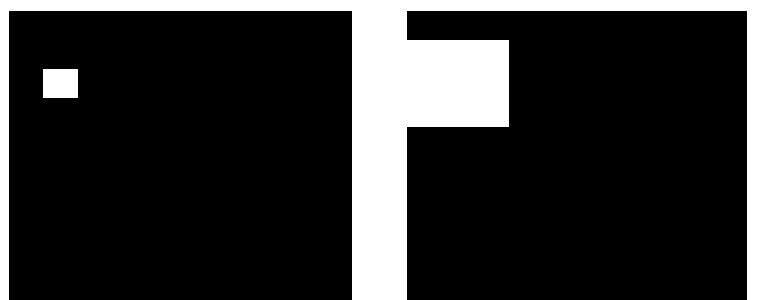

Fig.4. Before (left) and after(right) dilation

The idea behind grayscale dilation is similar to binary with the exception of the way in which the kernel related with the input image is derived. In that case, these kernels are 3-D rather than 2-D.

Grayscale dilation with a structuring element normally brights the image. Bright regions bounded by dark regions enlarge in size, besides dark regions bounded by bright regions compress in size. Minor dark spots in images vanish as those are filled in by surrounded intensity. Small bright spots becomes larger. Fig.5 shows a vertical cross-section of a gray-level image and the effect of dilation using a disk shaped structuring element.

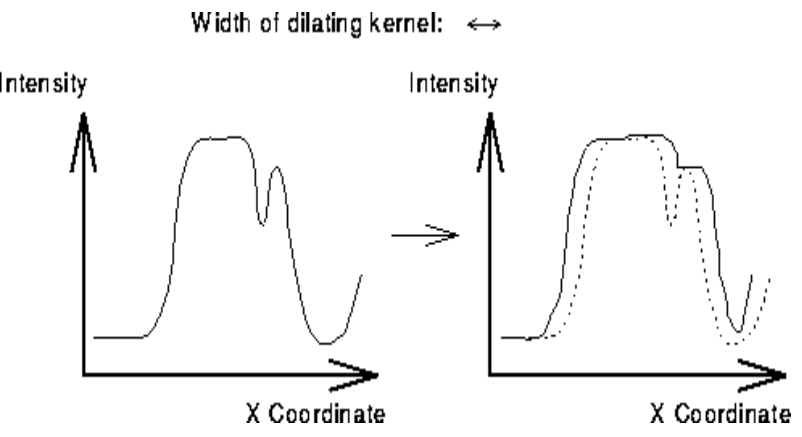

Fig.5. A cross-section (vertical) through a gray level image 


\section{B. Erosion}

As like dilation, erosion operator requires two input data. One is the image and another is a (typically small) kernel or a structuring element. It is this structuring element makes the effect of the erosion on the image. Fig. 2 depicts how erosion works using same structuring element of fig2.

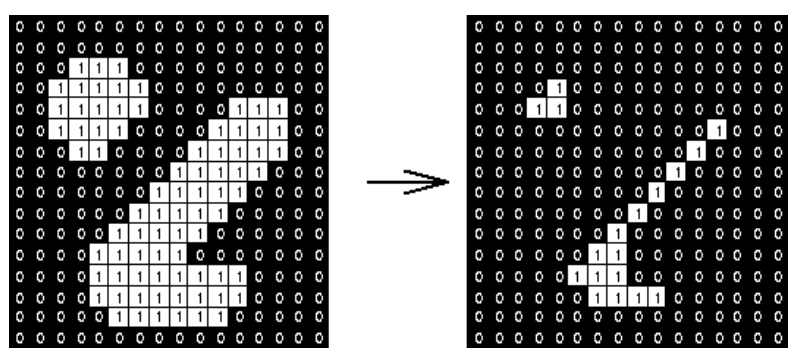

Fig.6. Effect of erosion

\section{Opening}

In simple form, opening refers to erosion which is followed by way of dilation keeping the same kernel or structuring element.. Like dilation, opening operator also demands two inputs. One is the image (to apply opening operation) and another is the kernel or structuring.

Similarly, gray level opening involves gray level erosion followed by gray level dilation. Effect of opening operation is shown in fig.7 as an example. Fig. 8 shows effect of opening on a binary image.
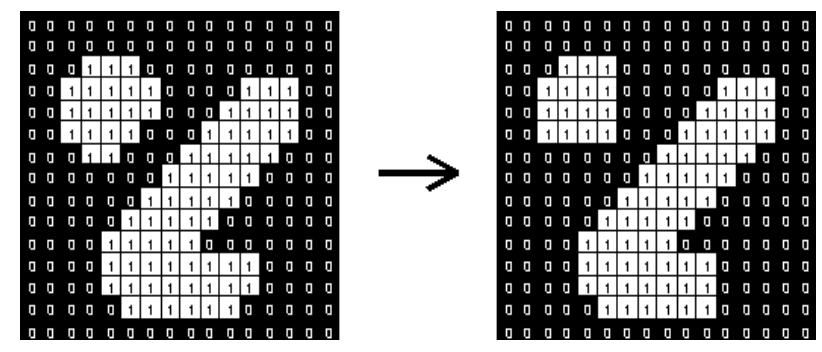

Fig.7. Opening operation
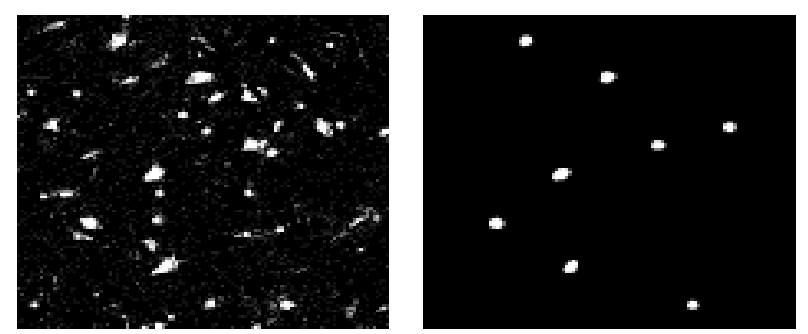

Fig.8. before (left) and after (right) opening

\section{Detection}

Detection of breast cancer is usually means to identify cancer cell regions. In this system mammogram images are considered to detect breast cancer. Mammogram images are suitable to detect breast cancer through image processing technique. Cancer infected regions are dense white pixels in mammogram image. The proposed system initially enhanced the input image by gamma correction. Fig.9(a) is the input image and fig.9(b) is the gamma correction. This gamma correction step is very useful for further processing. It makes the input image more flexible to detect cancer. Then the image is converted to gray. A very popular and common morphological technique named dilation is applied on the gray image. Dilated output is displayed in fig.9(c). 


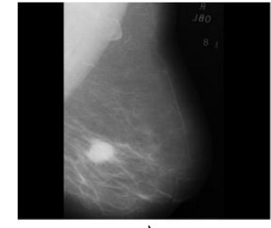

a)

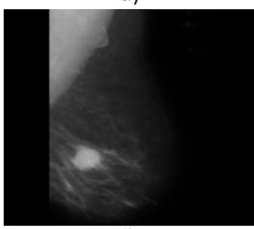

d)

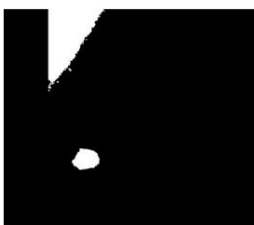

g)

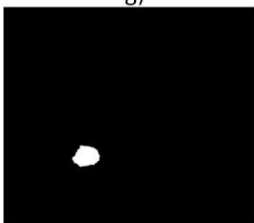

j)

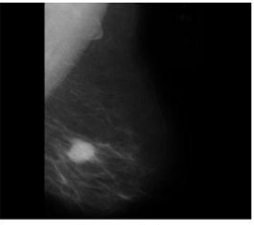

b)

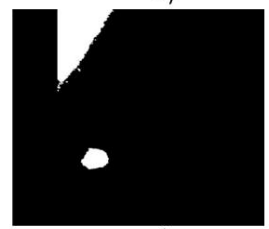

e)

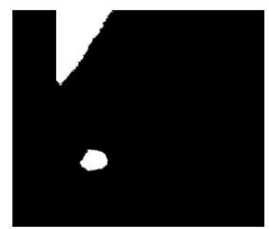

h)

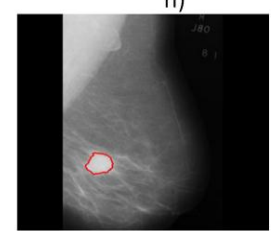

k)
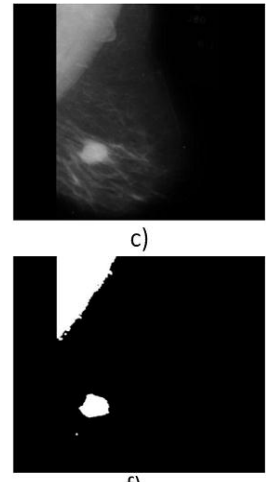

f)

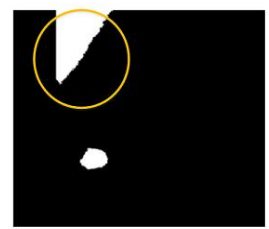

i)

Fig.9. Results of breast cancer detection steps a) input image b)gamma corrected image c) dilated image d) output of opening on dilated image e) binarization of dilated image f) binarization of opening image g) AND output h) small area filtered image i)hole filled image j)removed unwanted and desired roi $\mathrm{k}$ )marked detected region

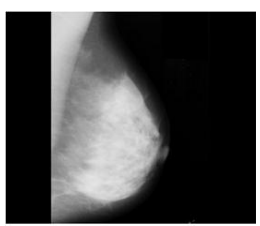

a)

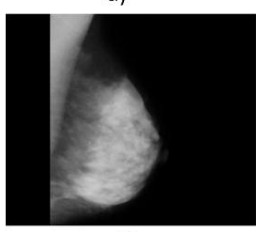

d)

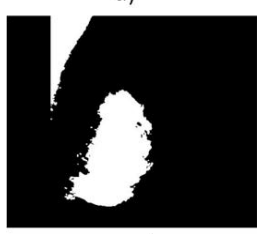

g)

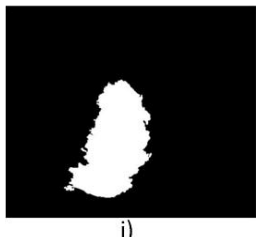

j)

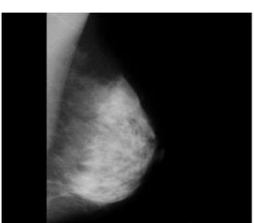

b)

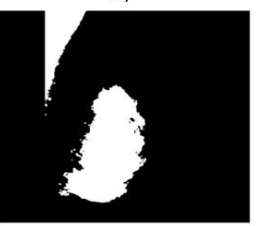

e)

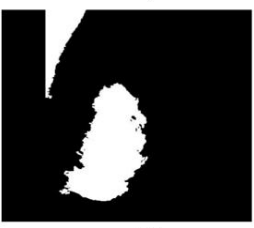

h)

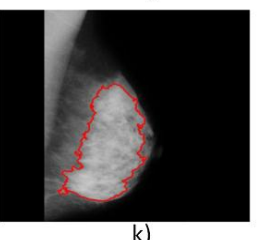

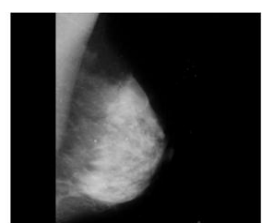

c)

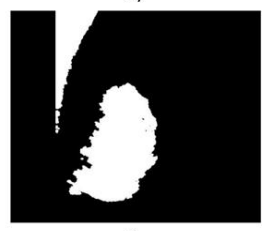

f)

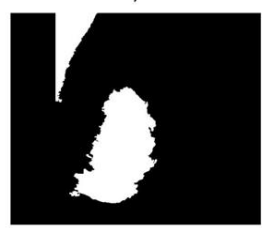

i)

Fig.10. Results of breast cancer detection steps a) input image b)gamma corrected image c) dilated image d) output of opening on dilated image e) binarization of dilated image f) binarization of opening image g) AND output $\mathrm{h}$ ) small area filtered image i)hole filled image j)removed unwanted and desired roi k)marked detected region 
Dilation is the way of expanding white pixels and reducing black pixels. It increases foreground area while decreasing background. After that opening operation is formed again on the dilated image. Principle behind opening technique is erosion task followed by dilation. Fig.9(d) shows result of opening operation. Later output of dilated image and opening image is transformed into binary image. Result shown in fig.9(e) and fig.9(f) respectively. An AND operation is applied between the two binary image. Thus it keeps the common pixels and removes other. Output of AND operation contains cell region and some noises. AND output displayed in fig.9(g). Small area based filtering is then employed on AND output. Then holes are filled. Fig.9(h) fig.9(i) are the area filtered and hole filling output. An unwanted object is usually exists at the top. Object at top position of image is considered as unwanted object. . First object returned by bounding box is usually unwanted object considered in this study. The object is circled in fig.9(i). Finally, that object is removed using bounding box parameter. Result is in fig.9(j) and it is the detected breast cancer region. A visualization of detected region is marked on input image. It is shown in fig.9(k). Few examples are also shown in fig.10 and fig.11.

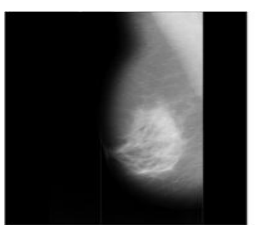

a)

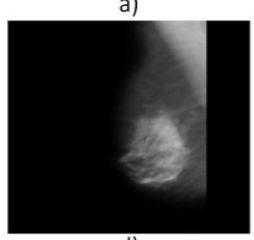

d)

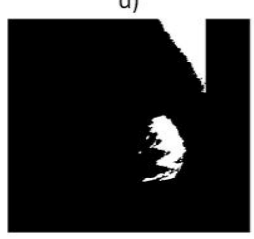

g)

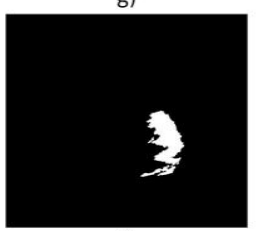

j)

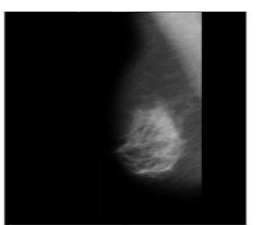

b)

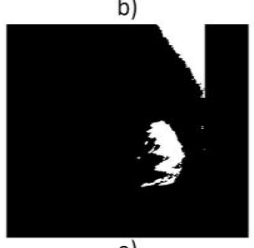

e)

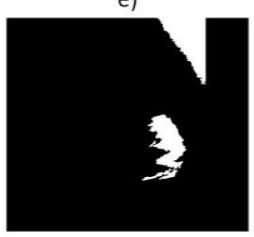

h)

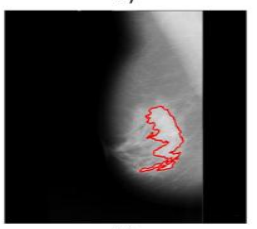

k)
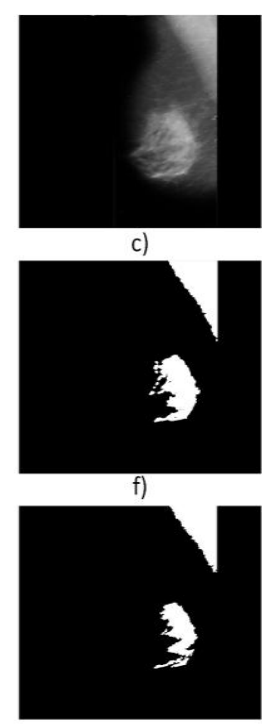

i)

Fig.11. Results of breast cancer detection steps a) input image b)gamma corrected image c) dilated image d) output of opening on dilated image e) binarization of dilated image f) binarization of opening image g) AND output $h$ ) small area filtered image i)hole filled image j)removed unwanted and desired roi k)marked detected region

\section{Conclusion and Future Work}

Every year a significant number of women are dies for the reason of breast cancer. Mammogram image depicts well output of breast scanning. Early detection of this cancer is hardly needed to reduce death and also risk of treatment. This detection method can be helpful in medical field. A computerized system can check for cancer regions within short period of time. This paper elucidates a method for breast cancer detection from mammogram images. Multiple morphological operation is processed in this study which results satisfactory. Gamma correction of the image proves better for additional processing. It also has a good effect in detecting cancer. However various issues are yet to solve for more better performance like contrast enhancement, segmentation and also standard dataset is also a issue. Combination of different methods will be analyzed in future as hybrid technique to get more efficiency and much reliability in detection of breast cancer. 


\section{References}

[1] Radiologyinfo.org,-Mammography, URL: https://www.radiologyinfo.org/en/info.cfm?pg=mammo\#overview

[2] Lisa Hutchinson, -Breast cancer: Challenges, controversies, breakthroughsl, Nature Reviews Clinical Oncology, Vol. (7), pp. 669- 670, December 2010. Doi:10.1038/nrclinonc.2010.192.

[3] Alasdair McAndrew, -An Introduction to Digital Image Processing with Matlab Notes for SCM2511 Image Processing 1\|, School of Computer Science and Mathematics Victoria University of Technology, Ch. 7, pp. 137-141, Semester 1, 2004.

[4] J. W. Xu, T. D. Pham and X. Zhou, -A double thresholding method forcancer stem cell detection,\| 2011 7th International Symposium on Image and Signal Processing and Analysis (ISPA), Dubrovnik, Croatia, 4-6 Sept. 2011, pp. 695-699.

[5] http://santemedecine.commentcamarche.net/contents/cancer/13_lecancer-du-sein.php3\#les-statistiques-alarmantes-du-cancerdu-sein

[6] http://www.cancerscreening.nhs.uk/breastscreen/publications/breastscreening-french.pdf

[7] http://www.nhs.uk/translationfrench/Documents/Cancer_of_the_breast_female_French_FINAL.pdf

[8] http://www.europadonna.fr/

[9] Samir M. Badawy1., Alaa A. Hefnawy2, Hassan E. Zidan3, and Mohammed T. GadAllah, Breast Cancer Detection with Mammogram Segmentation: A Qualitative Study,(IJACSA) International Journal of Advanced Computer Science and Applications, Vol. 8, No. 10, 2017

[10] R. B. Dubey, S. Bhatia, M. Hanmandlu and S. Vasikarla, -Breast Cancer Segmentation Using Bacterial Foraging Algorithm, 2013 10th International Conference on Information Technology: New Generations, Las Vegas, NV, 2013, pp. 541-545. Doi: 10.1109/ITNG.2013.88.

[11] M. Mustafa, N. A. Omar Rashid and R. Samad, -BreAst Cancer Segmentation Based On GVF snake, 2014 IEEE Conference on Biomedical Engineering and Sciences (IECBES), Kuala Lumpur, 2014, pp. 928-931. Doi: 10.1109/IECBES.2014.7047647

[12] Abdul Qayyum and A. Basit, -Automatic breast segmentation and cancer detection via SVM in mammograms,\| 2016 International Conference on Emerging Technologies (ICET), Islamabad, 2016, pp. 1- 6. Doi: 10.1109/ICET.2016.7813261.

[13] B. K. Gayathri and P. Raajan, -A Survey of Breast Cancer Detection Based on Image Segmentation Techniques, 2016 International Conference on Computing Technologies and Intelligent Data Engineering (ICCTIDE'16), Kovilpatti, 2016, pp. 15.

[14] F. Eddaoudi, F. Regragui, A. Mahmoudi, N. Lamouri, Masses Detection Using SVM Classifier Based on Textures Analysis, Applied Mathematical Sciences, Vol. 5, no. 8, 367 - 379, 2011

[15] Wener Borges Sampaio, Edgar Moraes Diniz, Arist 'ofanes Correa Silva, Anselmo Cardoso De Paiva and Marcelo Gattass, Detection of Masses in Mammogram Images using cnn, Geostatistic Functions and svm, Computers in Biology and Medicine, vol. 41(8), pp. 653-664, (2011)।

[16] Nasseer M. Basheer and Mustafa H. Mohammed, Segmentation of Breast Masses in Digital Mammograms using Adaptive Median Filtering and Texture Analysis, Int. J. Recent Technol. Eng.(IJRTE), vol. 2(1), pp. 2277-3878, (2013).

[17] Shruti Dalmiya, Avijit Dasgupta and Soumya Kanti Datta, Application of Wavelet Based k-means Algorithm in Mammogram Segmentation, International Journal of Computer Applications, vol. 52(15), pp. 15-19, (2012)

[18] https://www.kaggle.com/kmader/miasmammography?select=all-mias

\section{Author's Profile}

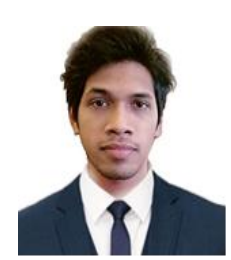

Prashengit Dhar received his B.Sc. degree in Computer Science and Engineering from University of Science and Technology Chittagong (USTC) and M.Sc. degree in Computer Science and Engineering from Port City International University. Currently he is working as a lecturer in a college. He has published many papers in conference and journal. His research interests include image processing, pattern recognition and machine learning.

How to cite this paper: Prashengit Dhar, " A Method to Detect Breast Cancer Based on Morphological Operation", International Journal of Education and Management Engineering (IJEME), Vol.11, No.2, pp. 25-31, 2021. DOI: 10.5815/ijeme.2021.02.03 